\title{
estudio técnico comparativo de puntales
}

JUAN MANUEL DE LA PENA AZNAR, Dr. Ingeniero Industrial

$851-1$

\section{sinopsis}

El autor del artículo describe y justifica técnicamente el tipo de puntal tubular telescópico ideado por él, consisiente en el UNICO puntal, que él conozca, en el mundo del doble pasador.

Para tal justificación el autor tiene forzosamente que desarrollar la teoría matemática de los puntales telescópicos, y aprovecha para acometer el sistema y difícil cálculo matemático de un puntal telescópico, habida cuenta de los dos tubos de diámetros y espesores diferentes de que se componen y de los huelgos internos y defectuoso asentamiento y apoyo de los puntales en las obras.

\section{PUNTAles METAlicos TElescopicos TUBULARES. TEORIA}

Hoy día, salvo casos muy particulares, es indiscutible el empleo de puntales metálicos telescópicos tubulares para sostener encofrados horizontales, y también para apuntalamiento oblicuo de encofrados de muro, así como para entibación de zanjas. Los puntales de madera maciza ya no se utilizan más que en obras pequeñas, tanto por su pequeña carga portante como por su pequeño número de reempleos.

Los puntales, cualquiera que sea su material, trabajan a pandeo, cuyo origen es el siguiente: Cuando se estudian piezas rectas sometidas a compresión y flexión simultáneas, se ve que existe un cierto valor de la fuerza compresora, denominada carga crítica, para la que puede producirse una gran flecha, aunque la carga transversal sea muy pequeña. Para una pieza prismática con los extremos articulados la carga crítica es:

$$
P_{c}=\frac{\pi^{2} \cdot E \cdot I}{l^{2}}
$$

Experimentalmente se ve que cuando la fuerza compresora de una pieza esbelta se aproxima a ese valor, comienza a curvarse la pieza, y esta deformación lateral aumenta con tal rapidez al crecer la compresión, que una carga igual a la crítica es suficiente para producir la ruina completa de la estructura.

De la expresión anterior se deduce, como hecho curioso, que la carga crítica no depende de la resistencia del material, sino solamente de las dimensiones de la pieza y del módulo de elasticidad del material. Así, dos piezas igualmente esbeltas, una de acero de alta resistencia y la otra de acero normal, pandean para el mismo valor de la fuerza compresora, aunque el material sea muy diferente en ambos casos.

También por la ecuación [1] se ve que puede aumentarse la resistencia a pandeo incrementando el momento de inercia $I$, lo que puede lograrse sin modificar el área de la sección recta, a base de distribuir el material tan lejos como sea posible de los ejes principales de la sección. Por esto, las secciones tubulares son las más aptas y económicas para barras comprimidas. Disminuyendo el espesor de la pared y aumentando las dimensiones transversales -en el caso del tubo, el diámetro exterior - se aumenta la estabilidad de las columnas tubulares. 
Existe, sin embargo, un límite inferior del espesor de la pared, el tubo, para el que dicha pared resulta inestable por sí misma, y en lugar de pandear la pieza como un todo, se presenta el pandeo de sus elementos locales, lo que origina la abolladura de la pared.

La tensión crítica de pandeo se obtiene dividiendo la fórmula [1] por el área de la sección recta $A$ de la columna:

$$
\sigma_{c}=\frac{P_{c}}{A}=\frac{\pi^{2} \cdot E}{\left(\frac{l}{i}\right)^{2}}
$$

observándose que para un material dado el valor de la tensión crítica depende del que toma la relación $l / i=\lambda$, denominada esbeltez. Para valores elevados de $\lambda$ la $\sigma_{c}$ es muy reducida, lo que indica que la columna esbelta pandea y pierde su resistencia con una tensión de compresión pura muy pequeña. A medida que la esbeltez disminuye, la tensión crítica aumenta, y la curva $A B C$ de la figura 1 se aproxima asintóticamente al eje vertical. Esta es la curva de Euler, que tiene un límite de empleo como veremos.

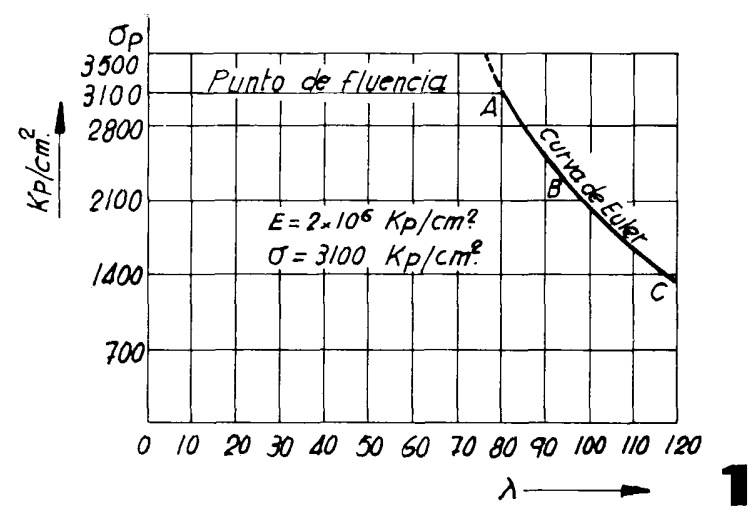

En efecto, de la expresión [1] de la carga crítica se ha obtenido una ecuación diferencial para la elástica, lo que supone que el material es perfectamente elástico y sigue la ley de Hooke. Por consiguiente, la curva $A B C$ de la figura 1 solamente da resultados satisfactorios para piezas esbeltas, en que $\sigma_{c}$ permanezca inferior al límite elástico del material.

En la figura 1 se da un resultado experimental. El acero es corriente con un acusado punto de fluencia $\sigma_{F}=3.100 \mathrm{kp} / \mathrm{cm}^{2}$. Se ve que para columnas de gran esbeltez, $\lambda>80$, el valor que da el ensayo para $\sigma_{P}$ coincide satisfactoriamente con la curva de Euler.

En el caso de puntales tubulares telescópicos, normalmente, el diámetro medio de los tubos está en torno a los $45 \mathrm{~mm}$, y como para los tubos

resulta que

$$
i \sim 0,35355 \mathrm{dm}(\mathrm{dm} \text { en } \mathrm{cm})
$$

$$
i \sim 1,6 \mathrm{~cm}
$$

Por otro lado, los puntales de obras normales, cerrados, tienen una altura de unos $2 \mathrm{~m}$ y abiertos de unos $3 \mathrm{~m}$, así que los puntales telescópicos tubulares trabajan con esbelteces que van de

$$
\lambda_{\min }=\frac{200}{1,6}=125 \quad \text { a } \quad \lambda_{\max }=\frac{300}{1,6}=187,5,
$$

luego tales puntales trabajan siempre en el campo elástico de la curva de Euler. 
Aunque los aceros empleados en los tubos para puntales tienen un límite de fluencia bajo, $\sigma_{F} \sim 2.400 \mathrm{kp} / \mathrm{cm}^{2}$, y a veces menos, el límite de proporcionalidad es aún más bajo, por lo que la curva de Euler es solamente válida para esbelteces $\lambda>100$, y ya hemos visto que los puntales tubulares siempre trabajan $\operatorname{con} \lambda>100$.

Profundizando más en el tema descubrimos que no existe la columna o puntal perfectos, pues todos adolecen de una excentricidad intrínseca de fabricación; otra de colocación, y, por fin, la última de trabajo, ya que raro es el puntal que asiente correctamente en la obra.

Por eso Sidebottom ha estudiado el caso del sólido que posea una curva de deformación compuesta de dos rectas (fig. 2), con módulos de elasticidad $E$ desde el origen 0 hasta $\sigma_{E}$, y otra $\alpha^{*} E$, para $\alpha^{*}<1$. Los resultados obtenidos, en cuanto a la carga máxima soportada $\sigma_{c}=P_{c} / A$, se dan en función de $\alpha^{*}$ y de la excentricidad inicial $e: h$ en la figura 3 , cuyas ordenadas son la relación $\sigma_{c} / \sigma_{E}$ y las abscisas la variable $\varepsilon_{E} \cdot \lambda^{2}$, siendo $\varepsilon_{E}=\sigma_{E} / E$ y $\lambda=l / i$,

o sea, la esbeltez.
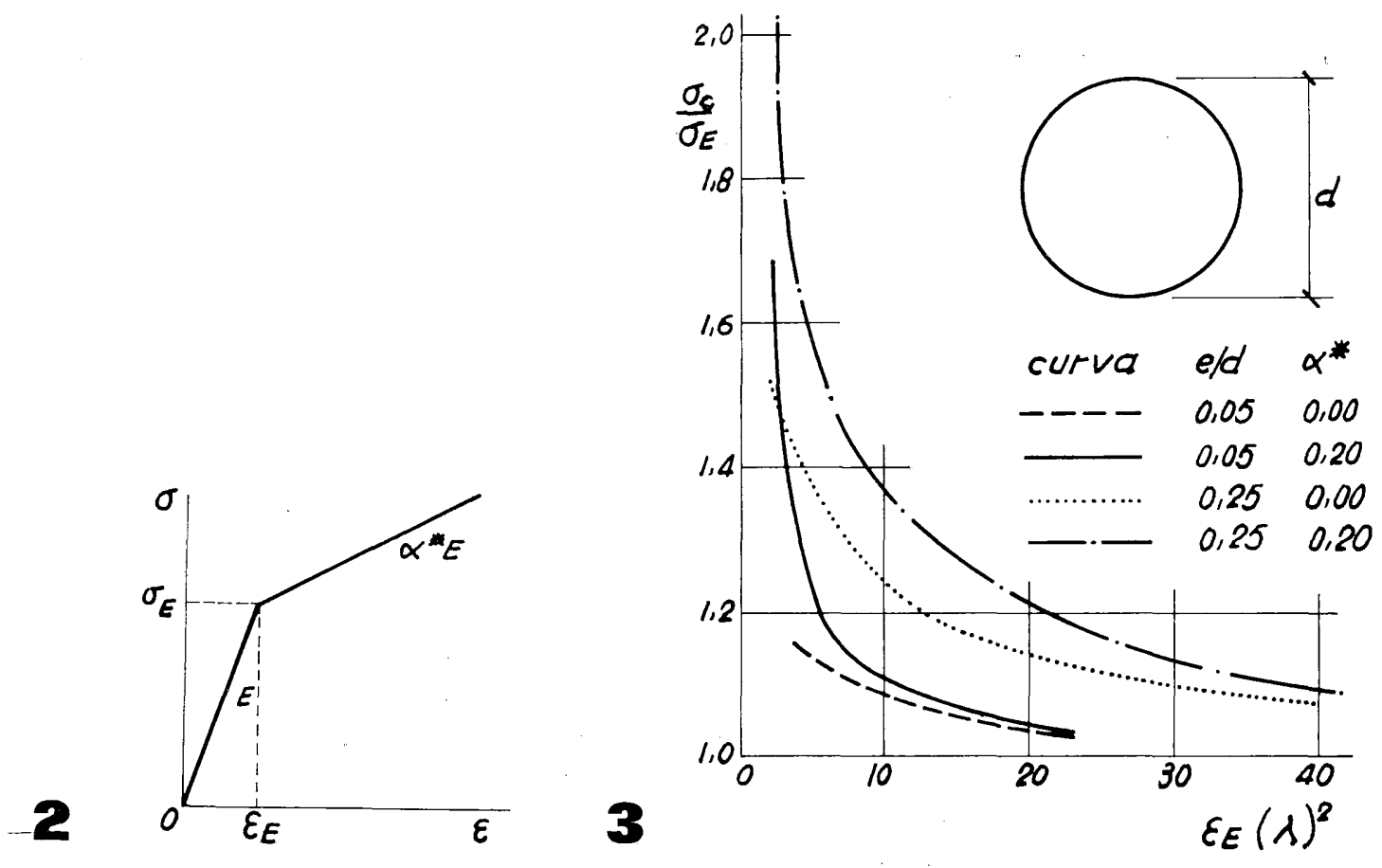

Los espesores $e$ se han relacionado con el diámetro para dos casos extremos: $e / d=0,05$, por ejemplo un tubo normal de puntal con $\sim 50 \mathrm{~mm} \varnothing$ y $e \sim 2,5 \mathrm{~mm}$, y $e / d=0,25$ para tubos de conducción de pared gruesa tal como $25 \mathrm{~mm}$ y un $\varnothing$ de $100 \mathrm{~mm}$.

Obsérvese en la figura 3 la influencia que tiene $\alpha^{*}$, que mide la excentricidad, afectando sensiblemente a las curvas que ligan $\sigma_{C} / \sigma_{E} \operatorname{con} \varepsilon_{E} \cdot \lambda^{2}$.

Toda esta teoría, que acabamos de exponer, ha sido la base tomada por el autor para idear un nuevo tipo de puntal con doble pasador, único en el mundo que él sepa, que con el mismo peso de materiales, fundamentalmente tubos, soporta a pandeo el doble que todos los demás que usan un solo pasador y tubos más gruesos o con mayores espesores. 
Volviendo a lo expresado en la figura 3, para que se vea cuán importante es el problema de la excentricidad, pues raro es el puntal que en obra apoya su base correctamente, así como la superior, si está rematado por igual pieza, en la figura 4 se esquematiza la más desfavorable posición de trabajo del puntal con excentricidad $e$ y el consiguiente gráfico 1 de cargas $P$ a pandeo y las flechas $f$ producidas para cada una.

Pues bien: en Alemania se toma como carga de trabajo $P$ de puntales, con excentricidad de carga $P$ a (ancho de base) la siguiente fórmula:

$$
P=\frac{3 \cdot L_{\max }}{L^{2}}
$$

siendo $L$ la luz de pandeo en cada caso, y $L_{\max }$ la mayor altura que puede alcanzar el puntal de que se trate.

\section{TIPOS Y CARACTERISTICAS}

De los catálogos editados por los diversos fabricantes nacionales que ha podido consultar el autor, he aquí el cuadro número 1, resumen de los tipos y características de los puntales telescópicos existentes en el mercado español:

\section{U A D R O 1}

\begin{tabular}{|c|c|c|c|c|c|c|c|c|}
\hline \multirow{2}{*}{ FABRICAN'TE } & \multirow{2}{*}{ TIPO } & \multirow{2}{*}{$\begin{array}{l}\text { Altura } \\
\text { mínima } \\
(\mathrm{m})\end{array}$} & \multirow{2}{*}{$\begin{array}{c}\text { Carga } \\
\underset{\left(C_{\max }=\mathrm{kp}\right)}{ }\end{array}$} & \multirow{2}{*}{$\begin{array}{l}\text { Altura } \\
\text { máxima } \\
(\mathrm{m})\end{array}$} & \multirow{2}{*}{$\begin{array}{c}\text { Carga } \\
\text { minima } \\
\left(C_{\min }=\mathrm{kp}\right)\end{array}$} & \multirow{2}{*}{$\begin{array}{c}\text { Peso } \\
(P=\mathrm{kp})\end{array}$} & \multicolumn{2}{|c|}{$\begin{array}{l}\text { CARACTERISTICAS } \\
\text { ESPECIFICAS }\end{array}$} \\
\hline & & & & & & & $C_{\max }: P$ & $C_{\min }: P$ \\
\hline $\begin{array}{llllll}\text { ALL-FIX } & \ldots & \ldots & \ldots & \ldots & \ldots\end{array}$ & II & 2,50 & 7.000 & 4,20 & 4.400 & 17,0 & 411,8 & 258,8 \\
\hline $\begin{array}{llllll}\text { BAGANT } & \ldots & \ldots & \ldots & \ldots & \ldots\end{array}$ & Normal I & 1,25 & 2.600 & 2,00 & 1.950 & 6,9 & 377,0 & 283,0 \\
\hline $\begin{array}{llllll}\text { BAGANT } & \ldots & \ldots & \ldots & \ldots & \ldots\end{array}$ & Normal II & 1,50 & 2.600 & 2,45 & 1.650 & 8,2 & 317,0 & 201,0 \\
\hline $\begin{array}{llllll}\text { BAGANT } & \ldots & \ldots & \ldots & \ldots & \ldots\end{array}$ & Normal III & 1,75 & 2.600 & 3,00 & 1.380 & 9,8 & 265,0 & 141,0 \\
\hline $\begin{array}{llllll}\text { BAGANT } & \ldots & \ldots & \ldots & \ldots & \ldots\end{array}$ & Normal IV & 2,00 & 2.600 & 3,40 & 1.300 & 10,6 & 245,0 & 123,0 \\
\hline $\begin{array}{llllll}\text { BAGANT } & \ldots & \ldots & \ldots & \ldots & \ldots\end{array}$ & Fuerte I & 1,50 & 2.600 & 2,45 & 2.450 & 10,8 & 241,0 & 227,0 \\
\hline $\begin{array}{llllll}\text { BAGANT } & \ldots & \ldots & \ldots & \ldots & \ldots\end{array}$ & Fuerte II & 1,75 & 2.600 & 3,00 & 2.300 & 12,7 & 205,0 & 181,0 \\
\hline $\begin{array}{llllll}\text { BAGANT } & \ldots & \ldots & \ldots & \ldots & \ldots\end{array}$ & Fuerte III & 2,00 & 2.600 & 3,40 & 1.700 & 13,9 & 187,0 & 122,0 \\
\hline $\begin{array}{llllll}\text { BAGANT } & \ldots & \ldots & \ldots & \ldots & \ldots\end{array}$ & Fuerte IV & 2,50 & 2.600 & 4,40 & 1.650 & 19,2 & 135,0 & 86,0 \\
\hline JJEIP (Talleres ULMA). & Fuerte & 2,10 & 9.400 & 3,65 & 4.700 & 16.5 & 569,7 & 248,8 \\
\hline JJEIP (Talleres ULMA). & Fuerte & 3,65 & 5.000 & 5,25 & 2.300 & 21,5 & 232,5 & 106,9 \\
\hline KING (Tall. INGUNZA). & Reforzado & 2,00 & 5.250 & 4,50 & 1.750 & -- & - & - \\
\hline MILLS $\ldots \ldots \ldots \ldots \ldots$ & A & 1,10 & 8.500 & 1,83 & 7.900 & 14,7 & 578,2 & 537,4 \\
\hline MILLS $\ldots \ldots \ldots c c c c c$ & B & 1,75 & 8.500 & 3,12 & 4.900 & 20,9 & 406,7 & 234,4 \\
\hline MILLS $\ldots \ldots \ldots \ldots \ldots$ & $\mathrm{C}$ & 2,00 & 8.500 & 3,30 & 4.300 & 22,0 & 386,4 & 195,4 \\
\hline MILLS $\ldots \ldots \ldots \ldots \ldots$ & $\mathrm{D}$ & 2,60 & 8.500 & 4,00 & 3.500 & 25,0 & 340,0 & 140,0 \\
\hline MILLS $\ldots \ldots \ldots \ldots \ldots$ & $\mathrm{E}$ & 3,20 & 8.500 & 4,90 & 2.000 & 30,0 & 283,3 & 66,6 \\
\hline $\begin{array}{cccccc}\text { TAUXME } & (\text { Hnos. Quin- } \\
\operatorname{tana}) & \ldots & \ldots & \ldots & \ldots & \ldots\end{array}$ & $2,3 / 4,6$ & 2,30 & 5.910 & 4,60 & 1.530 & 13,0 & 454,6 & 117,7 \\
\hline TREGAR, S. A. ... ... ... & B I & 2,00 & 10.000 & 3,70 & 4.250 & 15.1 & 662,2 & 281,4 \\
\hline TREGAR, S. A. ... ... ... & B II & 2,50 & 10.000 & 4,20 & 3.750 & 16,6 & 602,4 & 225,9 \\
\hline TREGAR, S. A. ........ & B III & 3,10 & 6.250 & 4,70 & 3.000 & 18,1 & 345,3 & 165,7 \\
\hline TREGAR, S. A. ... ... ... & B IV & 4,00 & 4.000 & 5,70 & 2.500 & 21,4 & 186,9 & 116,8 \\
\hline URBINA $\ldots \ldots, \ldots, \ldots$ & Punto naranja & 2,80 & 3.600 & 5,00 & 2.200 & - & - & - \\
\hline $\begin{array}{llllll}\text { URBINA } & \ldots & \ldots & \ldots & \ldots & \ldots\end{array}$ & Punto naranja & 3,30 & 3.300 & 6,00 & 1.800 & - & - & - \\
\hline
\end{tabular}



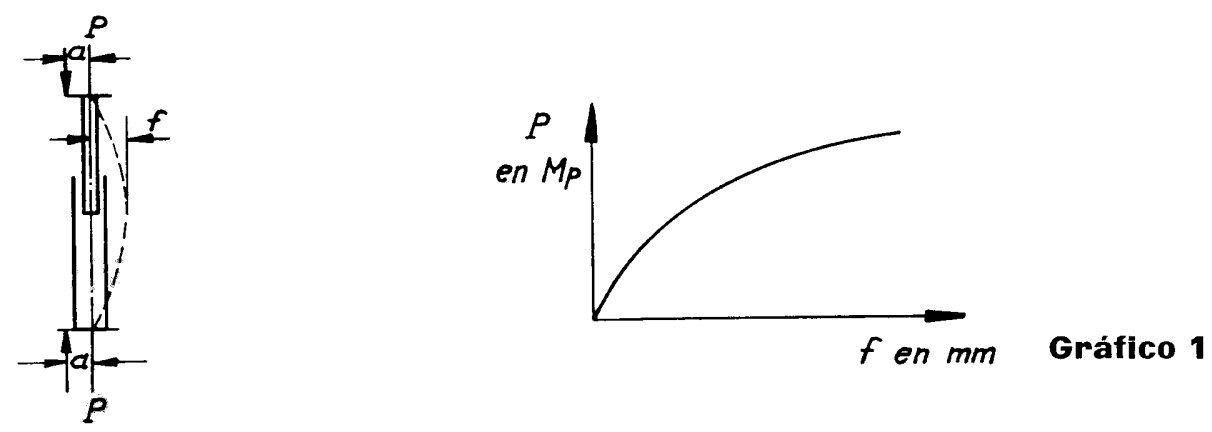

Sobre las características de los puntales, en definitiva la carga de trabajo y altura para la que pueda utilizarse esa carga hay bastante que hablar, pues por la comparación que haremos más adelante entre las curvas de rotura de los puntales expresados en el cuadro número 1, se verán no ya las diferencias entre ellos, sino, lo que es más incomprensible, las curvas de rotura de muchos de ellos, que, por no decirlo de otra manera, diremos que son "caprichosas», y esto que este tema de sustentación de cargas de encofrado es algo para tratarlo muy seriamente, y exigir una investigación oficial de cargas reales científicamente obtenida.

En los puntales se prevé que el usuario va a utilizar al máximo la capacidad portante de ellos, por lo que se deberán tomar dos importantes precauciones en su colocación: primera, inmovilizar tanto la cabeza como la base del puntal, para prevenir posibles desplazamientos sobre su empleo, y segunda, como la capacidad de carga depende de la esbeltez, se deberán colocar riostras intermedias horizontales y diagonales para reducir las luces libres en los puntales de gran longitud.

Frecuentemente el hormigonado se realiza por medio de carretillas a motor o con calderos, de forma que el hormigón se vierte con gran rapidez sobre una zona limitada del encofrado. La utilización de estos medios puede tener como consecuencia ciertos levantamientos temporales de los encofrados próximos a la zona donde se ha vertido el hormigón; si así ocurre, cabe la posibilidad de que esos encofrados se separen de los puntales que los soportan, y a menos que ambos se mantengan rígidamente unidos variarán sus posiciones relativas. Por el mismo motivo las bases de los puntales deberán estar convenientemente fijados a los durmientes. Muchos encofrados se han derrumbado debido a que, durante el hormigonado, los puntales se han desplazado de su posición inicial.

Volviendo a los datos facilitados por el cuadro número 1, se resaltan las dos últimas columnas, que vienen a dar una idea de la calidad del proyecto y construcción de cada tipo de puntal, puesto que esas columnas son los cocientes de las cargas máxima y mínima de trabajo, por el peso del puntal para cada luz de pandeo determinada.

Para hacer más expresivo el cuadro número 1 hemos trazado la figura 5 , que nos da la calidad de cada tipo de puntal medida por el cociente carga/peso, es decir, por el aprovechamiento de la materia del puntal para cada luz de pandeo.

Como, naturalmente, dentro de un modelo de una marca, por ejemplo el modelo $\mathrm{B}$ de la marca TREGAR, hay varios tipos, en este caso el B-I, B-II, B-III y B-IV, debido a que es imposible resolver con un solo tipo todas las alturas y cargas que se presentan en la realidad, los constructores, aún conservando como es lógico las características fundamentales de cada modelo, dividen éste en varios tipos, cada uno con su campo de aplicación entre dos alturas y cargas perfectamente definidas.

De ahí que el llevar a un gráfico las características específicas de cada tipo de puntal de una marca, para los diversos tipos existentes dentro de un mismo modelo se obtengan, como se 
observa en la figura 5, diversos puntos en función de la luz, que no están sobre la misma curva, y ésta hay que trazarla como el centro de gravedad de los puntos hallados.

Se comprueba por dicha figura 5, cosa que era de esperar una vez estudiado el próximo epígrafe, "Puntal con doble pasador», que éste es el que mejor aprovecha la materia prima tubular de que se compone, como los demás puntales.

Luego su calidad, desde el punto de vista estrictamente tecnológico, le coloca en primera posición.

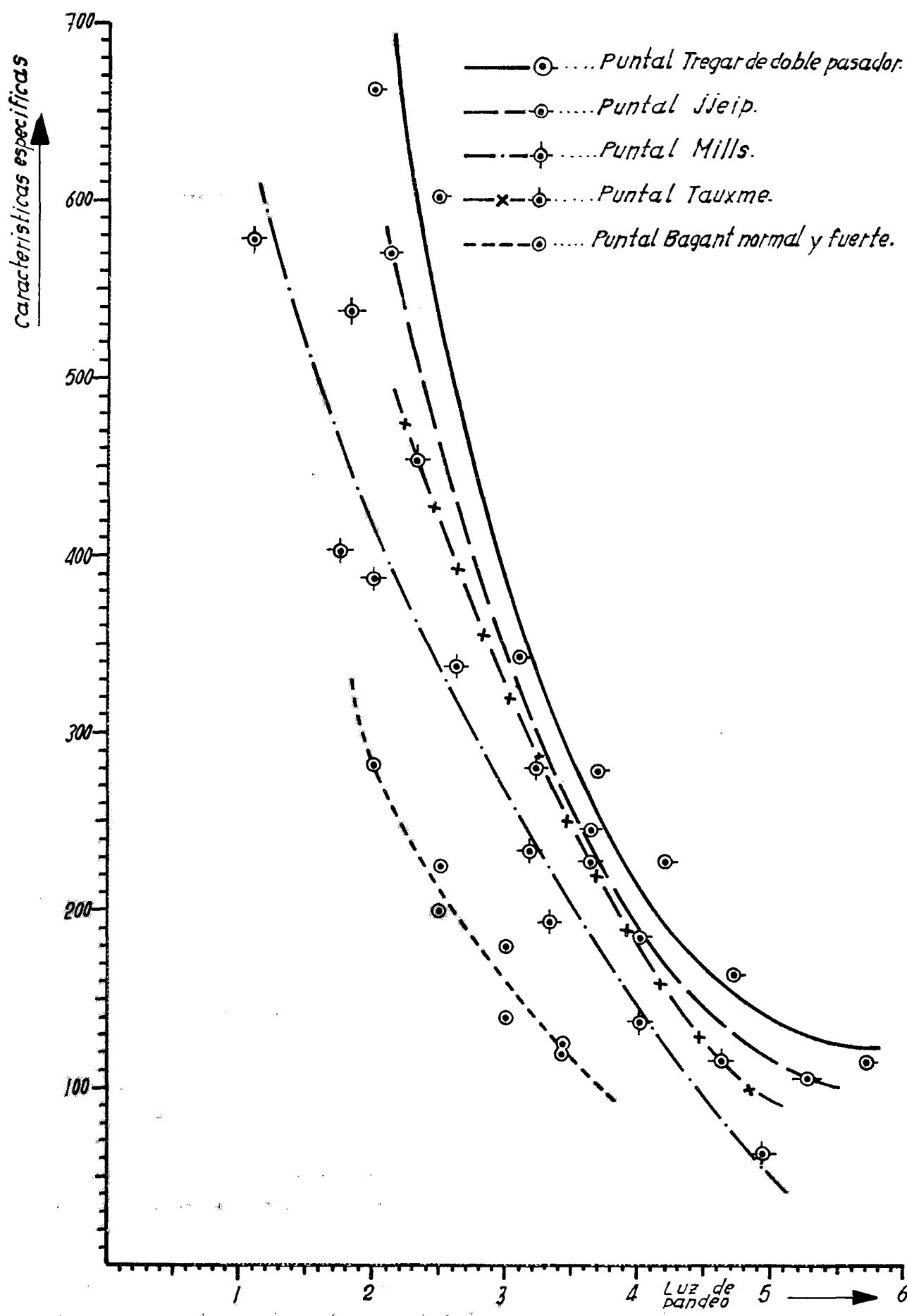




\section{PUNTAL CON DOBLE PASADOR}

Para obtener un puntal con el máximo de resistencia posible, sin aumentar su peso, y con menor esbeltez posible, para alcanzar la mayor estabilidad posible también, el autor ideó hace tiempo el puntal con doble pasador, cuyo principio es el siguiente:

Se sabe que el trabajo a pandeo de un puntal depende de su sebeltez, $\lambda$, la cual es un número abstracto cociente de dividir la luz de pandeo, $l$, por el radio de giro de la sección recta del material del puntal, $i$.

$$
\lambda=\frac{l}{i}
$$

Se sabe también que el radio de giro, $i$, de las secciones cilíndricas, o tubos que se emplean para materializar los puntales tubulares telescópicos, depende del diámetro medio de aquéllas, $d_{m}$, de su espesor, $e$, según la siguiente fórmula muy aproximada:

$$
\mathrm{i} \approx \sqrt{\frac{d_{m}^{2}+e^{2}}{8}}
$$

Como en la práctica los tubos que materializan los puntales telescópicos tienen una relación entre $d_{m}$ y e del orden de:

$$
d_{m} \approx 20 \cdot e
$$

luego se comete un error despreciable, por defecto, al simplificar la fórmula anterior que nos da $i$, introduciendo esta relación de $d_{m}$ y $e$ acabada de dar, de manera que:

$$
i \approx 0,35355 \cdot d_{m}
$$

De las fórmulas anteriores se deduce que, para aumentar la carga a pandeo de un puntal, es preciso elevar el diámetro medio de los tubos empleados sin aumentar su peso, $p$, o sea reduciendo su espesor, $e$, cuyos tres valores ligados por la expresión

$$
p=2,466 \cdot e \cdot d_{m} \mathrm{kp} / \mathrm{m}
$$

estando $e$ y $d_{m}$ en $\mathrm{cm}$.

Ahora bien: al disminuir el espesor, $e$, para mantener invariable el peso, $p$, o sea el coste, por el aumento conveniente del diámetro, $d_{m}$, para aumentar la carga a pandeo con igual precio de materia prima, es preciso evitar la aparición del fenómeno de la rasgadura de los taladros del tubo, y de aquí la novedad única del puntal ideado por el autor, que con su doble pasador posee cuatro puntos de apoyo entre los tubos telescópicos.

Por consiguiente, por la fórmula [4] el puntal ideado por el autor puede tener un espesor, $e$, mitad que el que necesitan los tubos de todos los demás puntales del mercado, que solamente emplean un solo pasador a igualdad de peso, $p$, ya que su doble pasador le proporciona cuatro puntos de apoyo, o sea, el doble de los que tienen un solo pasador.

Ahora bien: siendo el nuevo radio de giro, $i^{\prime}$, doble para este nuevo tipo de puntal, por la fórmula [3] la nueva esbeltez, $\lambda^{\prime}$, será la mitad para igual luz de pandeo, $l$, y siendo la tensión crítica, calculada por Euler:

$$
\sigma_{c}=\frac{\pi^{2} \cdot E}{\lambda^{2}}
$$

resulta que sustituyendo $\lambda$ por $\lambda^{\prime}$, siendo

$$
\lambda^{\prime}=\frac{\lambda}{2}
$$

$\sigma_{c}^{\prime}$, para el puntal de doble pasador, será cuatro veces superior que la de todos los demás puntales de un solo pasador de igualdad de peso de tubo, o sea, prácticamente de coste de materia prima del puntal. 
En la figura 6 quedan bien reflejadas las diferencias dimensionales, estructurales y conceptuales de este nuevo tipo de puntal con los de un solo pasador.

Consideraciones de orden práctico impiden alcanzar plenamente esa meta, pues no existen tubos con espesores tan sutiles como los requeridos por esta nueva técnica del doble pasador.

En la práctica, los diámetros de los tubos empleados por el autor en puntales de doble pasador son 1,5 vecf:s de los demás tipos concurrentes de uno solo, con lo que su tensión crítica a pandeo es dos veces superior (teóricamente, 2,25), a igualdad de peso y prácticamente costo del tubo empleadr.

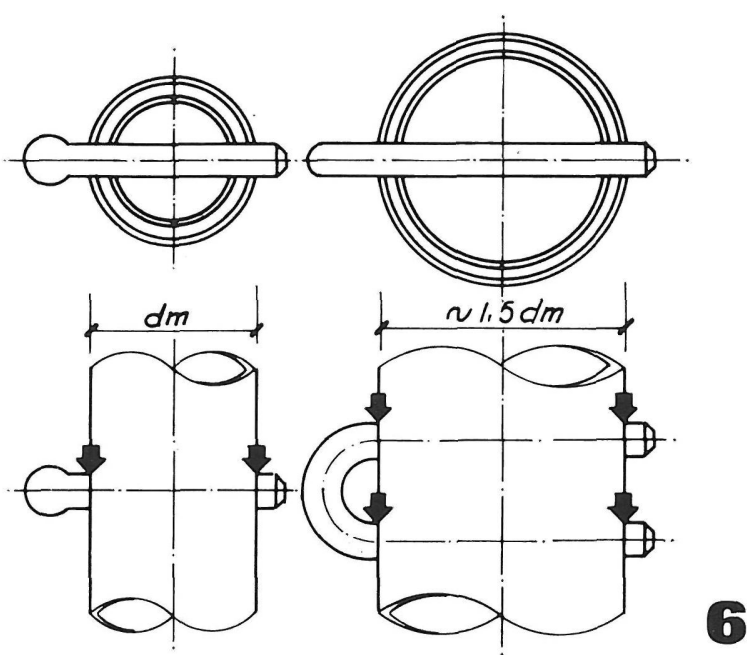

\section{CAlCUlo de UN PUNTAl TELEScopico}

Consideremos una barra de longitud $l$ (fig. 7), cuyas extremidades están articuladas, pero aseguradas en todas direcciones para que tal barra permanezca en línea recta durante la deformación, consecuencia de la aplicación de una carga $P$ en su extremo superior. Si $P$ es menor que un cierto valor $P_{c}$ que denominamos carga crítica, el equilibrio será estable y una ligera deformación $\delta$ producida por una carga $Q$ transversal desaparecerá al eliminar la acción de $Q$.

Esta carga crítica, $P_{c}$, puede calcularse con ayuda de ecuaciones simplificadas de la flexión, para lo cual supongamos dos secciones muy próximas, $A B$ y $F M C D$, de una barra flectada según la figura 8 , en la que como consecuencia del momento flector exterior entre ambas secciones se ha producido un giro $d \varphi$.
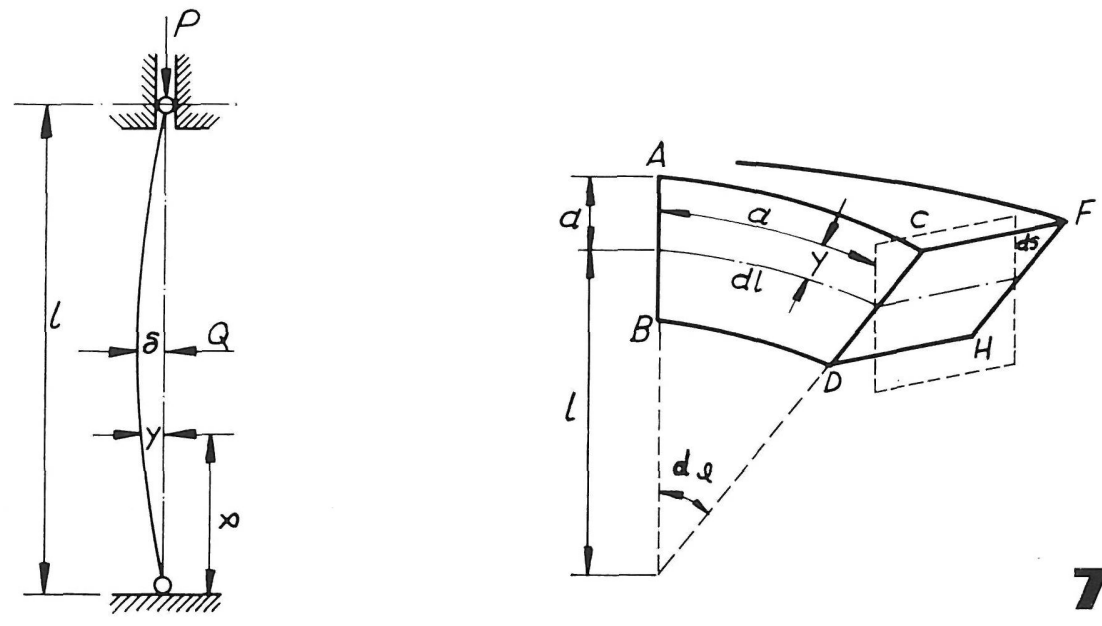

7 y 8 
La longitud de la fibra neutra $d l$ sigue siendo la misma, pero otras fibras, como, por ejemplo, la $a$ situada a $y$ por encima de esa línea neutra, ha aumentado de longitud. Vamos a establecer una relación entre la tensión $\sigma$ a esa distancia y de la línea neutra, donde está el elemento $d s$ de la sección girada $F H C D$, y su causa, el momento flector, para lo cual tomamos momentos, vale:

$$
M=\int \sigma d s \cdot y=\int E \cdot y^{2} \cdot \frac{d \varphi}{d l} \cdot d s=E \cdot \frac{d \varphi}{d l} \int y^{2} \cdot d s
$$

Pero $\int y^{2} \cdot d s$ es el momento de inercia de la sección respecto de la línea neutra, luego:

$$
M=E \cdot I \cdot \frac{d \varphi}{d l}
$$

Ahora bien: si el radio de giro medio de la pieza es $\varrho$ se verifica que $d l=\varrho \cdot d \varphi$ y sustituyendo en [5] resulta

$$
\frac{1}{\varrho}=\frac{M}{E \cdot I}
$$

que traducida al lenguaje nos dice que la curvatura que adopta una pieza sometida a flexión es directamente proporcional al momento flector, e inversamente proporcional al momento de inercia de la pieza.

Por otra parte, la expresión del radio de curvatura de una pieza flectada es:

y su inversa:

$$
\varrho=\frac{\left[1+\left(\frac{d y}{d x}\right)^{2}\right]^{3 / 2}}{\frac{d^{2} y}{d x^{2}}}
$$

$$
\frac{1}{\varrho}=\frac{\frac{d^{2} y}{d x^{2}}}{\left[1+\left(\frac{d y}{d x}\right)^{2}\right]^{3 / 2}}
$$

Pero el denominador de [7] es muy cercano a 1, porque las deformaciones son muy pequeñas $\mathrm{y}(d y / d x)^{2} \approx 0$, luego

$$
\frac{1}{\varrho}=\frac{d^{2} y}{d x^{2}}=\frac{M}{E \cdot I}
$$

en la que $y$ es la flecha del punto de coordenada $x$ (fig. 7).

Tomando momentos en la figura 7 de las fuerzas exteriores, respecto del punto $x, y$, tendremos:

$$
M=-P \cdot y-\frac{Q}{2} \cdot x
$$

Luego la ecuación [6] se transforma en:

$$
E \cdot I \cdot \frac{d^{2} y}{d x^{2}}=-P \cdot y-\frac{Q}{2} \cdot x
$$

cuya integral es del tipo general siguiente:

siendo

$$
y=A \cdot \cos k x+B \cdot \operatorname{sen} k x-\frac{Q}{2} \cdot x
$$

$$
k^{2}=\frac{P}{E \cdot I}
$$


La determinación de las constantes $A$ y $B$ se realiza planteando las condiciones de tal ecua. ción, encontrándose finalmente que:

$$
y=\frac{Q \cdot l^{3}}{48 \cdot E \cdot I} \cdot 3 \cdot \frac{\operatorname{tg} u-u}{u^{3}}
$$

siendo:

$$
u=\frac{k \cdot l}{2}=\frac{l}{2} \cdot \sqrt{\frac{P}{E \cdot I}}
$$

Así, pues, la flecha es el producto de dos factores. El primero, $Q \cdot l^{3} / 48 \cdot E \cdot I$, que es la flecha obtenida por efecto de la fuerza transversal $Q$, siendo $P$ nula. Y el segundo, $3\left(\operatorname{tg} u-u / u^{3}\right)$, que no depende más que de $P$.

Así, cuando $P$ aumenta independientemente de $Q$, la flecha $y$ aumenta también y tiende al infinito para el valor de $u=\pi / 2$. En este caso, un esfuerzo $Q$ infinitamente pequeño es suficiente para que la flecha $y$ crezca indefinidamente y sobrevenga la ruina de la barra, aun cuando el infinitesimal esfuerzo $Q$ desaparezca.

La magnitud $u=\pi / 2$ nos conduce directamente a la determinación de $P_{c}$, cuya expresión no es otra que la encontrada por Euler, hace más de doscinetos años, por su propio método, y resulta ser:

$$
P_{c}=\frac{\pi^{2} \cdot E \cdot I}{l^{2}}
$$

Finalmente, la fórmula de Euler se puede escribir así:

$$
P_{c}=q_{0} \cdot \frac{\pi^{2} \cdot E \cdot I}{l^{2}}
$$

en la que:

$q_{0}=l$ cuando los extremos de la barra estén articulados.

$q_{0}=S^{2}$ cuando las dos extremidades estén articuladas y que $S$ - $l$ puntos de la barra repartidas uniformemente en su longitud están sujetos para permanecer en línea recta.

$q_{0}=2$ cuando un extremo esté empotrado y el otro articulado.

$q_{0}=1 / 4$ cuando un extremo esté empotrado y el otro libre.

$q_{0}=4$ cuando los dos extremos estén empotrados.

Pero por principio un puntal telescópico está formado por dos tubos que uno penetra en otro, luego tienen distinto $\varnothing$ y diferente $I$.

Sea la columna compuesta por dos partes de secciones diferentes de la figura 9. Sean $l_{1}$ y $l_{2}$ las longitudes de los dos trozos de que se compone tal columna e $I_{1}$ e $I_{2}$ sus momentos de inercia respectivos. Si $\delta$ es la flecha en el extremo libre, pues el inferior se supone empotrado, y $P$ la carga a que se somete dicha columna, los momentos producidos por aquella desviada $\delta$ en cada trozo serán:

$$
\begin{aligned}
& E \cdot I_{1} \cdot \frac{d^{2} y_{1}}{d x^{2}}=P\left(\delta-y_{1}\right) \\
& E \cdot I_{2} \cdot \frac{d^{2} y_{2}}{d x^{2}}=P\left(\delta-y_{2}\right)
\end{aligned}
$$


y haciendo

$$
P_{1}^{2}=\frac{P}{E \cdot I_{1}} \quad \mathrm{y} \quad P^{2}{ }_{2}=\frac{P}{E \cdot I_{2}}
$$

y teniendo en cuenta la condición de empotramiento de la base, la solución de esas ecuaciones [8] y [9] es:

$$
\begin{aligned}
& y_{1}=\delta+C \cdot \cos p_{1} \cdot x+D \cdot \operatorname{sen} p_{1} \cdot x \\
& y_{2}=\delta \cdot\left(l-\cos p_{2} \cdot x\right)
\end{aligned}
$$

Las constantes $C$ y $D$ se obtienen escribiendo $y_{1}=\delta$ para $x=l$; que $y_{1}=y_{2}$ para $x=l_{2}$, y que las deformadas de las dos curvas tienen la misma tangente en $x=l_{2}$, de donde:

$$
\begin{aligned}
& D=\frac{\delta \cdot \cos p_{2} \cdot l_{2} \cdot \cos p_{1} \cdot l}{\operatorname{sen} p_{1} \cdot l_{1}} \\
& C=-D \cdot \operatorname{tg} p_{1} \cdot l \\
& \operatorname{tg} p_{1} \cdot l_{1} \cdot \operatorname{tg} p_{2} \cdot l_{2}=\frac{P_{1}}{P_{2}} .
\end{aligned}
$$

Esta ecuación se puede resolver por aproximaciones sucesivas. Pero en el caso de los puntales telescópicos, ambas extremidades son articuladas, en la que hay que sustituir $l$ por $1 / 4$, y esto ha sido estudiado por Pflüger, dándose el esquema de cargas en la figura 10 , y los valores conducentes a la determinación de la carga crít:ca $P_{c}$ en la figura 7 .

$$
P_{c}=q_{0} \cdot \frac{\pi^{2} \cdot E \cdot I_{2}}{l^{2}}
$$

Timoshenko ha resuelto este caso por el método energético, a fin de obtener resultados aproximados.

Apliquemos los valores de la figura 10 al caso particular del puntal TREGAR tipo B-IV, que para simplificar cálculos supondremos $l_{1}=l_{2}=2 \mathrm{~m}$ y $l=l_{1}+l_{2}=4 \mathrm{~m}$ de luz de pandeo.
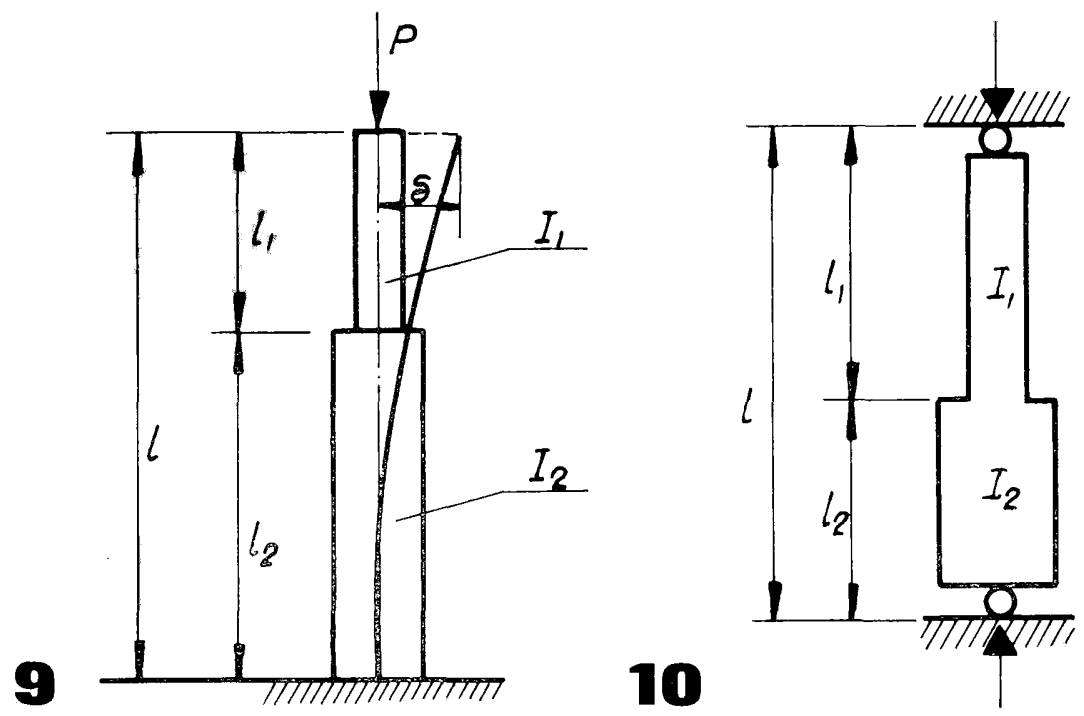
El tubo superior es de $\varnothing 61,5 \times 2,2 \mathrm{~mm}$, cuyo $I_{1}=18,04 \mathrm{~cm}^{4}$, y el tubo inferior es de $\varnothing$ $71,5 \times 1,8 \mathrm{~mm}$, cuyo $I_{2}=23,95 \mathrm{~cm}^{2}$, de modo que:

y

$$
i=\sqrt{\frac{I_{2}}{I_{1}}}=1,15
$$

$$
l_{1} / l=0,5
$$

Extrapolando en la figura 11 resulta que $q_{0} \sim 1,3$, luego

$$
P_{c}=1,3 \cdot \frac{\pi^{2} \cdot E \cdot 23,95}{400^{2}}=4.033 \mathrm{kp}
$$

y por la figura 13 podrá comprobarse que un putal TREGAR de $4 \mathrm{~m}$ de luz de pandeo, ensayado en el Instituto Torroja, dio una carga crítica o de ruina de $4.000 \mathrm{kp}$, lo que demuestra lo correcto del cálculo.

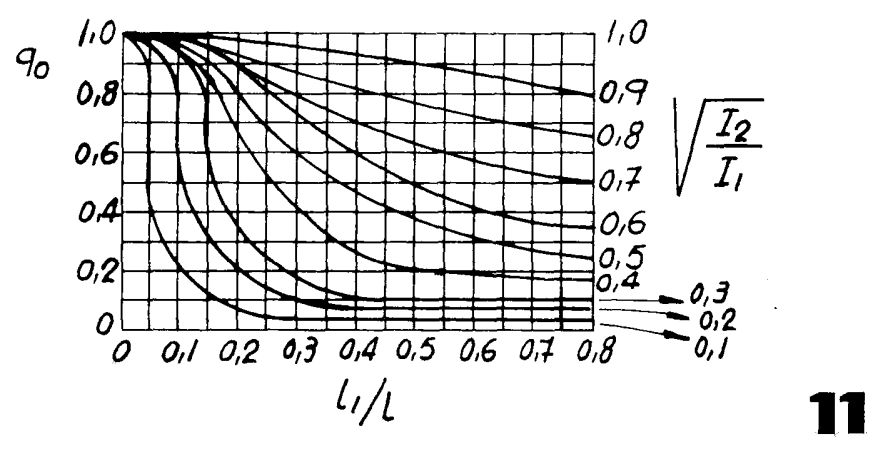

\section{COMPARACION ENTRE DIVERSOS TIPOS DE PUNTALES}

Decíamos en el apartado 1, al apuntar la Teoría de los puntales metálicos tubulares telescópicos, que trabajan siempre en el campo elástico de la curva de Euler, pues su esbeltez de trabajo oscila entre 125 y casi 200 , y que para los aceros empleados en la fabricación de los tubos usados en nuestro país, en los puntales, la curva de Euler sólo es válida para esbelteces superiores a 100 .

Por ello, partiendo de los datos facilitados por los diversos fabricantes nacionales, hemos confeccionado la figura 12 , en la que hemos dibujado las curvas de rotura de cada marca y modelo de puntal, en función de la luz de pandeo.

En el transparente situado encima de tal figura 12 hemos diseñado la hipérbola perfecta de Euler, y el lector podrá ver cuáles son las curvas, cuyos datos repetimos que los hemos obtenido de los catálogos de los fabricantes, se acercan más a la hipérbola teórica.

Pero el lector aún verá más, y es que ciertas marcas dan datos que llevados a la figura 12 citada dan por resultado hasta iiuna recta!!, o curvas que ni se parecen a la hipérbola teórica, a la que deben acercarse.

Esto no tiene más que dos explicaciones:

1. ${ }^{\text {a }}$ Que los datos dados en tales catálogos son erróneos, y cuyo origen del error ignoramos.

2. Lo que es peor, que tales datos no han sido obtenidos en laboratorio científicamente, luego no son fiables.

Hay algunas curvas de algunas marcas, que las hemos tenido que trazar con sólo dos puntos o datos, y si las hemos dibujado de curvatura parecida a la hipérbola teórica, lo hemos hecho a nuestra iniciativa, porque con ese número de datos es imposible trazar una curva real en esos casos. 


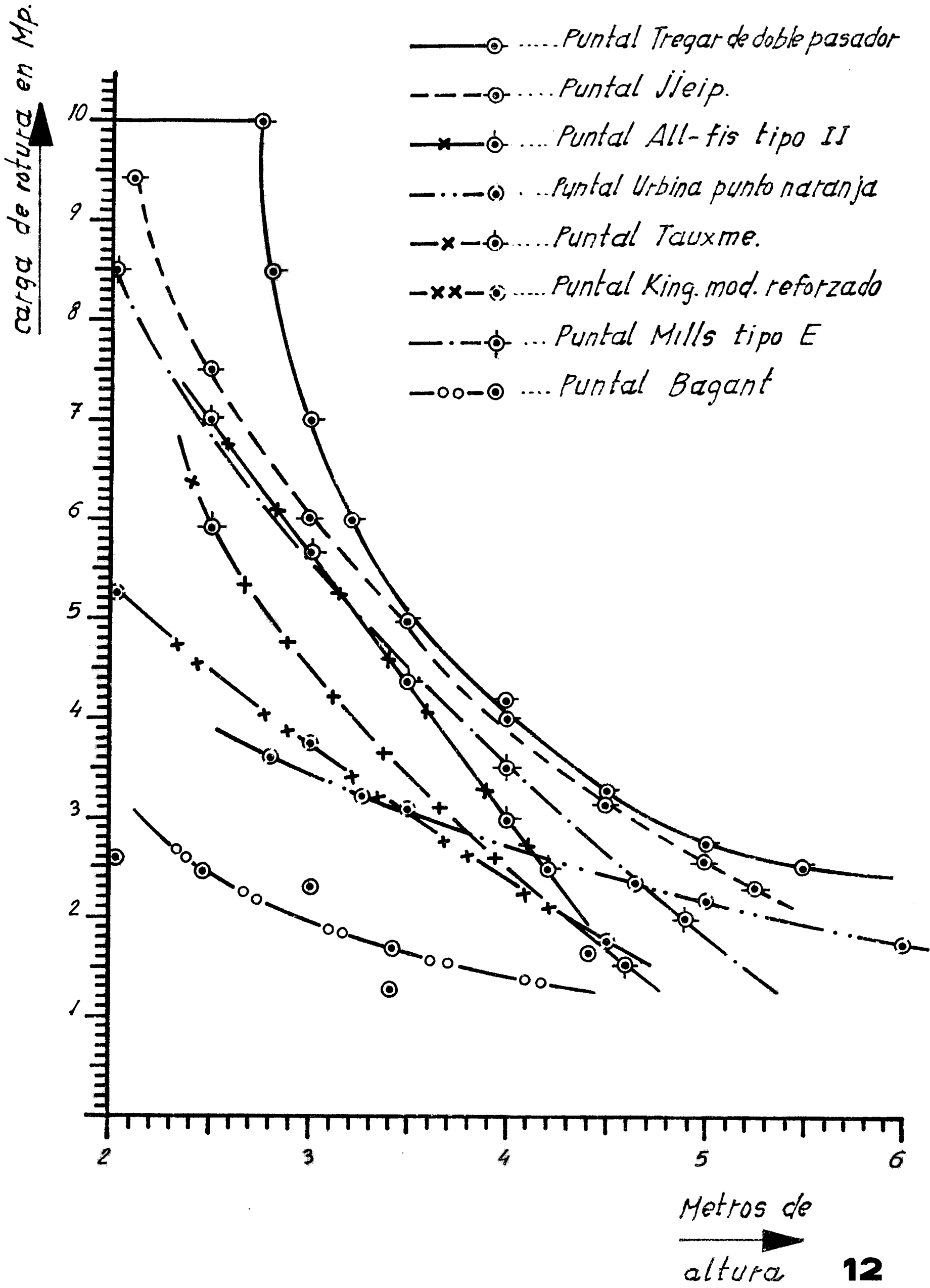




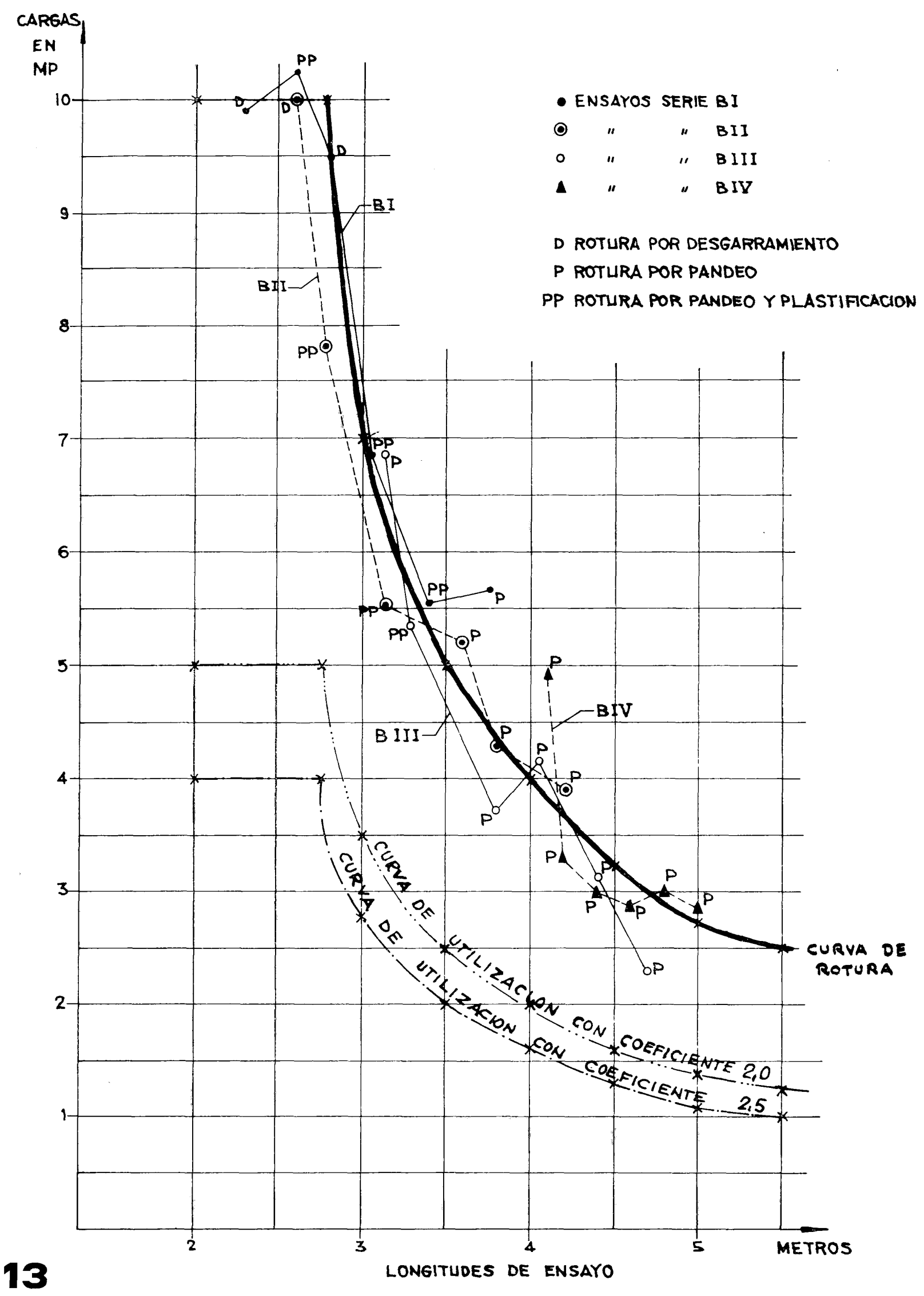




\section{CARGAS DE UTILIZACION. COEFICIENTE DE SEGURIDAD}

Hasta ahora todo lo dicho y calculado para puntales telescópicos tubulares, se refiere a la determinación de las cargas críticas de pandeo, pero en la realidad se ha de trabajar con cargas de seguridad, obtenidas a partir de $P_{c}$ dividiendo ésta por el coeficiente de seguridad $\nu$ que se adopte. Ello queda un tanto a la responsabilidad del usuario, quien podrá disminuir $v$ tanto como dedique atención al correcto y seguro montaje de los puntales.

Todo esto tiene mucha importancia para aprovechar al máximo la capacidad portante segura de un puntal, pues ya hemos visto que éstos trabajan con esbelteces superiores a 100, para los cuales vale la hipérbola de Euler y esta fórmula [1] nos dice que al aumentar al doble la longitud libre de pandeo, la carga admisible disminuye a la cuarta parte.

Por eso, en la figura 13 de la curva de cargas críticas de un tipo de puntal, como es el TREGAR, por ejemplo, hemos dibujado tres curvas de cargas de utilización para coeficientes de seguridad $\nu=2$; a 2,5 y a 3 .

Pero para usar puntales con el menor coeficiente de seguridad posible, no sólo deben estar aquéllos cuidadosamente colocados y asegurados al encofrado que soportan, sino también debidamente entretenidos, pues en principio los puntales telescópicos tubulares pueden usarse infinidad de veces, a condición de darles los debidos cuidados, especialmente en las roscas, engrasándolas periódicamente y pintándolas cuando el estado de su superficie lo requiera.

\section{résumé}

Etude technique comparative des étais

Juan Manuel de la Peña Aznar, Dr. ingénieur industrie!

L'auteur de cet article décrit et justifie techniquement le type d'étai tubulaire téles. copique conçu par lui-même. II s'agit du seul étai à double goujon, qu'il connaisse dans le monde.

Pour cette justification, l'auteur doit forcément développer la théorie mathématique des étais télescopiques et profite de cette cccasion pour aborder le système et le calcul mathématique difficile d'un étai télescopique, compte tenu des deux tubes ayant copique, compte tenu des deuxètres et des épaisseurs differents des diamètres et des épaisseurs differents
dont il se compose et des jeux internes et dont il se compose et des jeux internes et
de l'assiette et de l'appui défectueux des étais à pied d'oeuvre.

\section{summary}

Comparative technical study of pillars

Juan Manuel de la Peña Aznar, Dr. of Industrial Engineering

The author of this article technically describes and justifies the type of telescopic tubular pillar he created, which consists of the only pillar in the world which has a dcuble pin, as far as he knows.

In order to carry out this justification, the author is forced to develop a technical mathematical theory for telescoping pillars and he takes advantage of this opportunity to discuss the system and the difficult mathe discuss the system and the difficult mathebearing in mind that the two pipes involved bearing in mind that the two pipes involved
have different diameters and thicknesses, have different diameters and thicknesses,
the internal gaps and defective seating as well as the pillar support on the jobsite.

\section{zusammenfassung}

Technische vergleichsstudie für Verstrebungen

Dr. Juan Manuel de la Pena Aznar, Maschineningenieur

Der Autor des Artikels beschreibt und beweist in technischer Hinsicht die von ihm erfundene Type einer rohrförmigen, teleskopischen Verstrebung, die nach seinem Wissen die einzige Vertrebung mit Doppelriegel der Welt ist.

Für einen solchen Beweis muss der Auto zwangsläufig die mathematische Theorie der teleskopischen Verstrebungen enter und er nutzt die Gelegneheit, um das System und die komplizierte mathematische Berechnung einer teleskopischen Verstrebung auf Grund der zwei Rohre verschiedeung auf Grund der zwei Rohre verschiedeDicke, aus welchen eine solche basteht, und auf Grund der inneren Lücken und des mangelhalten Sitzes und Abstützung der Verstrebungen bei den Bauten darzustellen. 